\title{
Oxidation of Co-Based Porous Nanoparticles Followed by HAADF/BF imaging
}

\author{
Rubén Mendoza-Cruz and Francisco Ascencio-Aguirre
}

Universidad Nacional Autónoma de México, Mexico City, Mexico

Cobalt-based nanoparticles, in the form of pure and oxidized phase, exhibit interesting magnetic, catalytic, and transport properties that have motivated their production and the fundamental study. Cobalt monoxide $(\mathrm{CoO})$ crystallizes in a face-centered cubic (FCC) rock-salt structure, and its metastable hexagonal wurtzite-type phase has also been synthesized [1]. CoO nanoparticles present superparamagnetism or lowtemperature ferromagnetism depending on their size and shape. On the other hand, $\mathrm{CoO}$ nanoparticles have demonstrated an excellent efficiency for the catalytic reaction for water splitting, which emerged as promise candidates to reduce the use of precious metals [2]. Moreover, incorporating a second metal such as nickel has been beneficial for catalytic activity [3]. All the properties that nanoscale materials present are strongly dependent on their physical-chemical features, such as size, shape, structure, composition, ordering, assembly, etc., so the fundamental study of these characteristics enables the correlation with their displayed properties. In this regard, advances in scanning transmission electron microscopy (STEM) in high-angle annular dark-field (HAADF) and bright-field (BF) modes have boosted the structural characterization of nanoscale materials with sub-angstrom resolution. Particularly, the annular dark-field imaging has enabled the fast observation of materials' structural features taking advantage of the massthickness contrast. In transition-metal nanoparticles, such as iron, nickel, or cobalt, oxidation occurs when exposed to environmental conditions. An oxide layer is formed on the surface, and once it is formed, the metal core is further stabilized by this protective layer forming a core-shell configuration where the shell is typically a spinel oxide. In metal oxide systems, a phase with more metal content tends to be at the interior, while the phase with less content in metal grows on the surface. Hence, the study of the chemical and structural changes of nanoparticles is of paramount importance.

Herein, the oxidation of porous Co1-xNixO nanoparticles is followed by STEM imaging. The growth of a spinel Co3O4-type phase layer on the particles' surface was induced by the interaction with the electron beam to study the oxidation evolution of preformed CoO-type nanoparticles. Co1-xNixO nanoparticles were produced from a $\mathrm{Co}(\mathrm{ac})$ precursor thermally decomposed to form nanoparticles with a diameter of $\sim 50 \mathrm{~nm}$. Figure 1a corresponds to a low-magnification HAADF image of the as-synthesized nanoparticles. The HAADF imaging enabled observing these nanoparticles' porous structures, with cavities of less than $5 \mathrm{~nm}$ in diameter formed by gas release during growth in solution. Their morphology corresponded to truncated octahedrons which expose (111) and small (100) facets, with some monoatomic steps. The particles crystallized into a homogeneous rock-salt structure of $\mathrm{CoO}$. A high-magnification image is presented in Figure 1b. The chemical composition was calculated from energy dispersive spectroscopy (EDS), corresponding to a Co-rich oxide.

Oxidation of the CoO-type phase was induced by the interaction of these particles with the electron beam, enabling the observation of the chemical changes with atomic-level precision. It was observed that the growth of the spinel $\mathrm{Co} 3 \mathrm{O} 4$ phase started at the particle surface (Figure 1c). It possibly proceeds from the remaining oxygen in the microscope column and into the nanocavities, and the decomposition and segregation of the rock salt phase. However, this transformation was not observed in the cavities structure, suggesting a bubble-like structure of the nanoparticles. Furthermore, the chemical change was induced more rapidly at the corner sites of the nanoparticle than in the facets. The induced oxidation formed a core-shell configuration with a shell thickness of around 3-5 $\mathrm{nm}$, which apparently became stable for the observation conditions. 


\section{AKNOWLEDGMENTS}

R. Mendoza-Cruz thanks PAPIIT-UNAM for grant IA102020 to support this work.
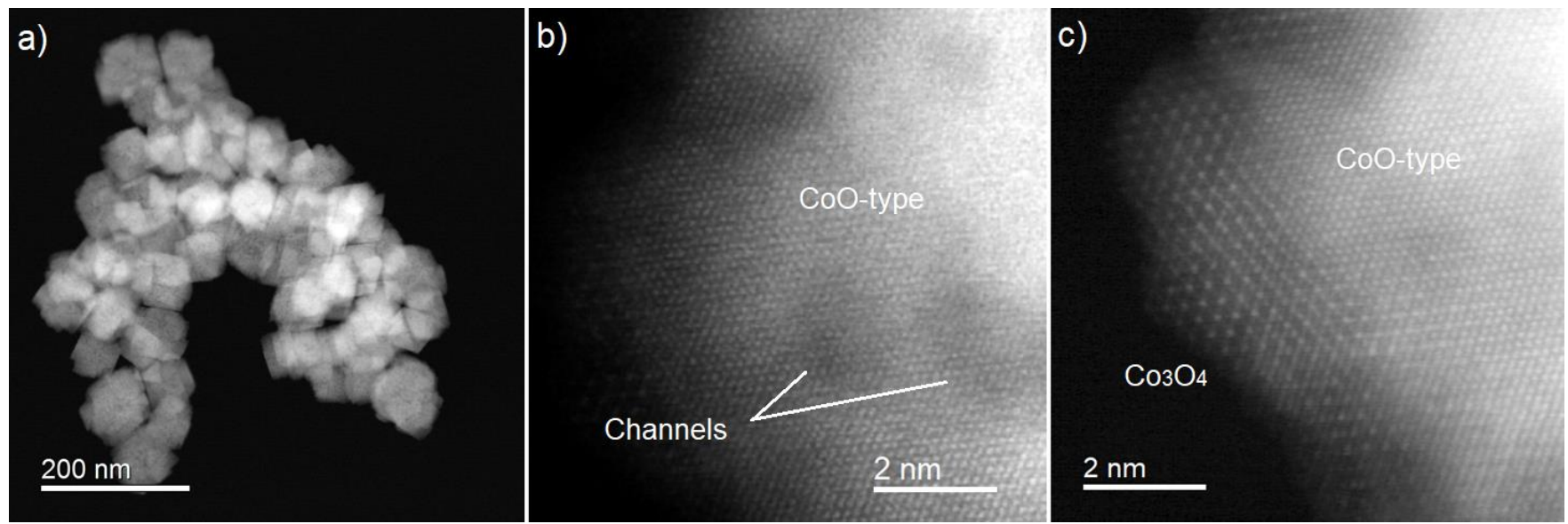

Figure 1. (a) Low magnification and (b) high-magnification HAADF image of the synthesized Co1-xNixO nanoparticles. (c) After exposure to the electron beam, the growth of a $\mathrm{Co} 3 \mathrm{O} 4$ surface layer is monitored.

\section{References}

[1] Nam KM, Shim JH, Han D-W, et al (2010) Syntheses and Characterization of Wurtzite CoO, Rocksalt $\mathrm{CoO}$, and Spinel Co $3 \mathrm{O} 4$ Nanocrystals: Their Interconversion and Tuning of Phase and Morphology. Chem Mater 22:4446-4454. https://doi.org/10.1021/cm101138h

[2] Liao L, Zhang Q, Su Z, et al (2014) Efficient solar water-splitting using a nanocrystalline CoO photocatalyst. Nat Nanotechnol 9:69-73. https://doi.org/10.1038/nnano.2013.272

[3] López-Tinoco J, Mendoza-Cruz R, Bazán-Díaz L, et al (2020) The preparation and characterization of $\mathrm{Co}-\mathrm{Ni}$ nanoparticles and the testing of a heterogenized $\mathrm{Co}-\mathrm{Ni} /$ alumina catalyst for co hydrogenation. Catalysts 10:. https://doi.org/10.3390/catal10010018 